\title{
Deficiency of Growth Factor Midkine Exacerbates Necrotizing Glomerular Injuries in Progressive Glomerulonephritis
}

\author{
Hiroshi Kojima, ${ }^{* \dagger}$ Tomoki Kosugi, $^{\dagger}$ Waichi Sato, ${ }^{\dagger}$ Yuka Sato, $^{* \dagger}$ Kayaho Maeda, $^{* \dagger}$ Noritoshi Kato, ${ }^{\dagger}$ Kiyonari Kato, ${ }^{\dagger}$ \\ Shinichiro Inaba, ${ }^{\dagger}$ Takuji Ishimoto, ${ }^{\dagger}$ Naotake Tsuboi, ${ }^{\dagger}$ Seiichi Matsuo, ${ }^{\dagger}$ Shoichi Maruyama, ${ }^{\dagger}$ Yukio Yuzawa ${ }^{\dagger \ddagger}$ and \\ Kenji Kadomatsu*
}

From the Departments of Biochemistry* and Nephrology, ${ }^{\dagger}$ Nagoya University Graduate School of Medicine, Nagoya; and the Department of Nephrology, ${ }^{\ddagger}$ Internal Medicine, Fujita Health University School of Medicine, Toyoake, Aichi, Japan

\author{
Accepted for publication \\ October 16, 2012. \\ Address correspondence to \\ Kenji Kadomatsu, Ph.D., M.D., \\ Department of Biochemistry, \\ Nagoya University Graduate \\ School of Medicine, 65 \\ Tsurumai-cho, Showa-ku, \\ Nagoya 466-8550, Japan. \\ E-mail: kkadoma@med. \\ nagoya-u.ac.jp.
}

\begin{abstract}
Inflammatory cell infiltration and fibrin deposition play important roles in the development of crescentic glomerulonephritis (GN). In particular, activation of coagulation is an indispensable factor in crescent formation. However, the mechanisms underlying the pathogenesis of crescent formation have not been completely elucidated. We identified the growth factor midkine (MK) as a novel key molecule in the progression of crescentic GN induced by anti-glomerular basement membrane antibody. Despite the lack of significant differences in autologous and heterologous reactions, MK-deficient $\left(\mathrm{Mdk}^{-/}\right)$ mice unexpectedly showed a greater number of necrotizing glomerular injuries than wild-type $\left(M_{d k^{+/+}}\right)$ mice. Likewise, more tubulointerstitial damage was observed in $\mathrm{Mdk}^{-/-}$mice, and this damage positively correlated with glomerular injury. Plasminogen activator inhibitor (PAI)-1 was strongly induced in the injured glomerulus of $\mathrm{Mdk}^{-/-}$mice, particularly in crescents and endothelial cells. This enhanced PAI-1 production was associated with an increase in inflammatory cell infiltration and matrix deposition in the glomerulus and the interstitium of $\mathrm{Mdk}^{-/-}$mice. In line with these in vivo data, primary cultured endothelial cells derived from $\mathrm{Mdk}^{-{ }^{-}-}$mice exhibited higher PAI-1 mRNA expression on fibrin challenge and less fibrinolysis than $M d k^{+/+}$mice. In contrast, the expression of plasminogen activators was not affected. Our combined data suggest that MK leads to a blockade of PAI-1, which is closely associated with the suppression of crescentic GN. (Am J Pathol 2013, 182: 410-419; http://dx.doi.org/10.1016/ j.ajpath.2012.10.016)
\end{abstract}

Crescent formation in the glomerulus develops as a result of segmental breaks of the glomerular basement membrane (GBM), often in association with fibrinoid necrosis. ${ }^{1}$ This component includes fibrin deposition, infiltration of inflammatory cells, and the extracellular matrix accumulation. Of these components, fibrin deposition by itself not only interrupts glomerular blood flow, leading to irreversible ischemia and glomerular obsolescence, but also promotes the infiltration of inflammatory cells and the proliferation of epithelial cells in Bowman's space. ${ }^{2}$ The recruitment of macrophages and neutrophils, which is triggered by inflammatory mediators such as fibrin, oxidative stress, and various chemokines, further leads to the activation of coagulation during crescentic glomerulonephritis (GN) and to thrombotic microangiopathy that is accompanied by severe endothelial dysfunction. ${ }^{1}$ It is therefore critical to break the vicious cycle of these processes to improve the mortality associated with aggressive kidney diseases.

The glycoprotein plasminogen activator inhibitor (PAI)-1, which is a serine protease inhibitor, is the major physiologic inhibitor of important plasminogen activators, such as

\footnotetext{
Supported in part by a Grant-in-Aid for Progressive Renal Diseases Research, Research on Intractable Disease, from the Ministry of Health, Labor, and Welfare of Japan and from the Ministry of Education, Culture, Sports, Science, and Technology (MEXT) of Japan (grant 22590886 to W.S. and grant 19590947 to Y.Y), by a Grant-in-Aid for Scientific Research on Innovative Areas from MEXT (grant 23110002 to K.K.), by Grants-in-Aid from MEXT (grant 20390092 to K.K.), and by funds from the Global Center of Excellence program, MEXT, to Nagoya University.
} 
tissue-type plasminogen activator (tPA) and urokinase-type plasminogen activator (uPA). ${ }^{3}$ Plasma and tissue concentrations of PAI- 1 are extremely low under physiologic conditions but increase in pathologic states. The roles of PAI-I are diverse, ranging from the development of thrombotic disorders to involvement in ischemic diseases, fibrotic disorders, metabolic syndrome, diabetes mellitus, and cancer. ${ }^{1,3,4}$ Many elegant studies suggest that up-regulation of PAI-1 in response to various stimuli, including high ambient glucose exposure, ${ }^{5}$ transforming growth factor (TGF)- $\beta{ }^{6}{ }^{6}$ oxidative stress, ${ }^{7}$ and angiotensin II, ${ }^{8,9}$ promotes interstitial macrophage recruitment and exerts direct cellular effects through binding to the uPA receptor. Indeed, PAI-1 deficiency reduces the severity of anti-GBM-induced nephritis. ${ }^{10}$ An understanding of the PAI-1-mediated signaling pathways involved in these processes might aid in the development of novel therapeutic strategies for rapid progressive GN.

Midkine (MK; alias Mdk) is a multifunctional, heparinbinding growth factor with major biological roles, such as neuronal survival and differentiation, cancer development, and inflammation. ${ }^{11}$ The neuronal cytoprotective effects of MK have been demonstrated in various in vivo models, including retinal degeneration induced by constant light exposure, ${ }^{12}$ cerebral infarction, ${ }^{13}$ and ischemia-induced neuronal death. ${ }^{14,15}$ In addition, MK regulates hypertension in kidney-lung interactions through activation of the reninangiotensin system, which eventually leads to progressive renal failure with elevated angiotensin $\mathrm{II}^{16} \mathrm{MK}$ also exacerbates inflammation-related diseases, as revealed by in vivo models of diseases such as rheumatoid arthritis, ${ }^{17}$ arterial restenosis, ${ }^{18}$ ischemic reperfusion-induced renal injury, ${ }^{19}$ diabetic nephropathy, ${ }^{20,21}$ and postinfectious GN. ${ }^{22}$ An inflammatory microenvironment induces $\mathrm{MK}$, which in turn, induces macrophage recruitment both directly and indirectly through activation of monocyte chemoattractant protein (MCP)-1.

The molecular mechanisms that induce PAI-1 under physiologic and pathologic conditions have been extensively studied. However, to date, systems that inhibit PAI-1 induction remain unknown. It is known that MK enhances fibrinolytic activity through the suppression of PAI-1 in vascular endothelial cells. ${ }^{23,24}$ In the present study, we uncovered an unexpected and beneficial role of $\mathrm{MK}$ in crescentic GN by analysis of accelerated Masugi nephritis in mice.

\section{Materials and Methods}

\section{Animals and Experimental Design}

Mice deficient in the MK gene $(M d k)$ were generated as described previously. ${ }^{25}$ After backcrossing of $M d k^{+/-}$mice for 14 generations with $129 / \mathrm{SV}$ mice, $M d k^{+/-}$mice were matched with each other to generate $M d k^{+/+}$and $M d k^{-/-}$ mice, which were used in this study. Experiments were performed on 8- to 12-week-old male $M d k^{+/+}$or $M d k^{-/-}$ mice weighing 20 to $30 \mathrm{~g}$ The mice were housed under controlled environmental conditions and maintained with standard food and water.

A previously characterized model of accelerated Masugi nephritis in mice was used. ${ }^{26-28} \mathrm{In}$ brief, $M d k^{+/+}$and $M d k^{-/-}$ mice ( $n=7$ in each group) that were sedated by general anesthesia underwent a right subcapsular nephrectomy. As described in Figure 1A, mice were preimmunized subcutaneously with $180 \mu \mathrm{g}$ of rabbit IgG (Pierce, Rockford, IL) in Freund's adjuvant-complete (Sigma-Aldrich, St. Louis, MO) and 7 days later were treated intravenously with $20 \mu \mathrm{L}$ of rabbit anti-mouse GBM antibody (a gift from Prof. Yoshio Suzuki and Tadashi Nagamatsu, Meijo University). Mice were sacrificed at 3 and 7 days after injection of the anti-GBM antibody. The kidneys were removed for histologic and biochemical analysis. Blood samples were collected on each day of sacrifice. Blood urea nitrogen (BUN) and urinary albumin were measured as described previously. ${ }^{16,22}$ All of the animal experiments were performed in accordance with the animal experimentation guidelines of Nagoya University School of Medicine.

\section{Immunofluorescence Staining of Rabbit IgG, Mouse $\mathrm{IgG}$, Mouse C3, and Neutrophils in Glomeruli}

A kidney tissue sample was embedded in OCT compound and frozen in liquid nitrogen for immunofluorescence staining. Sections (4- $\mu \mathrm{m}$ thick) were cut using a cryostat and then fixed in acetone. The cryosections were stained with fluorescein isothiocyanate (FITC) goat anti-rabbit IgG antibody (Cappel, West Chester, PA), FITC-rabbit anti-mouse IgG antibody (Zymed, San Francisco, CA), and FITC-goat antimouse C3 antibody (Cappel).

The cryosections were also stained with a monoclonal rat anti-mouse neutrophil marker 7/4 (Cedarlane, Ontario, Canada), followed by its detection with FITC-goat anti-rat $\operatorname{IgG}$ (Cedarlane). Leukocytes positive for the $7 / 4$ marker were counted by examination of sections of 20 glomeruli per subject. The glomeruli analyzed were cut at their vascular pole and were analyzed in a blinded manner by two independent observers. ${ }^{22}$ Negative controls were performed by replacement of the primary antibody with species-matched antibody.

\section{Morphologic Assessment}

The removed kidneys were fixed in $4 \%$ paraformaldehyde, embedded in paraffin, and then cut into $4-\mu \mathrm{m}$ sections. The sections were stained with PAS for evaluation of the development of severe glomerular damage and tubulointerstitial injury. The number of injured glomeruli per total glomeruli of each subject was calculated and expressed as a percentage. ${ }^{29}$ Glomerular abnormalities included crescent formation, which was defined as two or more layers of cells visible in Bowman's space, and severe necrosis that affected $>50 \%$ of the glomerular tuft. ${ }^{30}$ The percentage of 
A

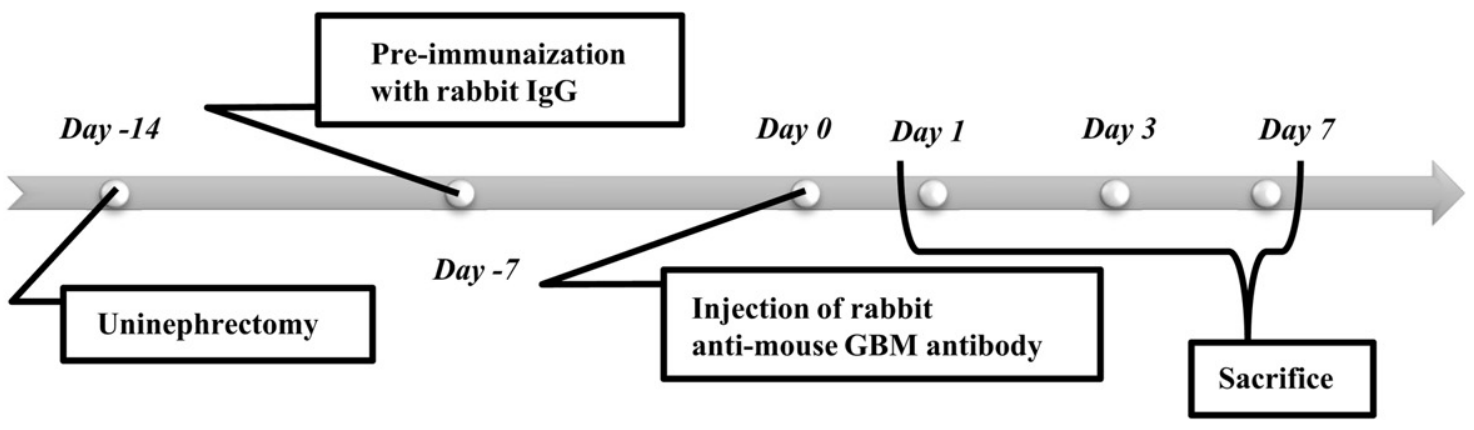

B
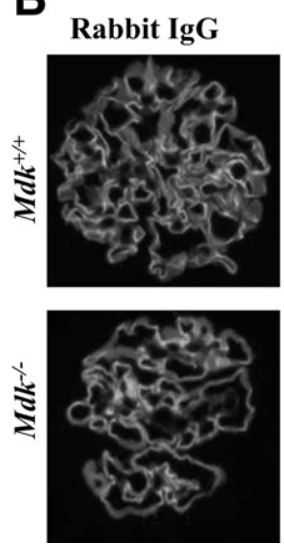

Mouse IgG
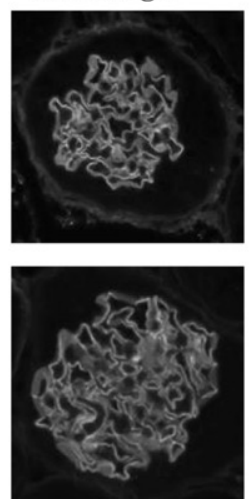

C
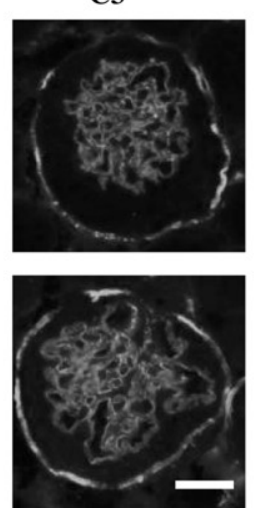
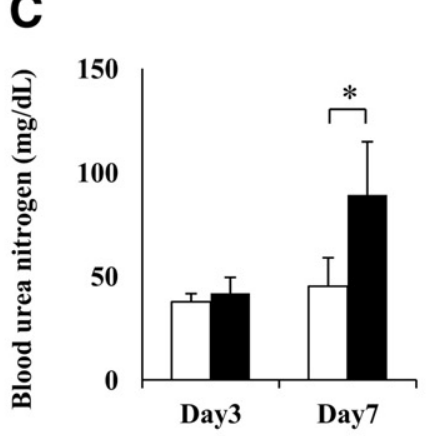

D

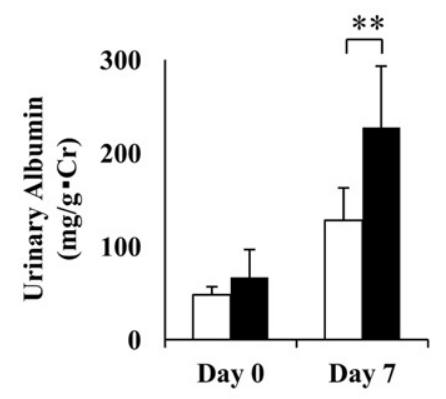

Figure 1 Experimental design, IgG and C3 deposition, and renal function. A: Experimental design. B: Immunofluorescence staining of rabbit IgG, mouse $\mathrm{IgG}$, and $\mathrm{C} 3$ on day 1 . Scale bar $=25 \mu \mathrm{m}$. C: BUN. D: Urinary albumin. Data are shown as mean (columns) and SD (bars). White bars, Mdk ${ }^{+/+}$mice; black bars, $\mathrm{Mdk}^{-1-}$ mice. ${ }^{*} P<0.01,{ }^{*} P<0.05 . n=7$ per group.

atrophic tubules (tubular dilation, detachment of tubular epithelial cells, condensation of tubular nuclei, and cast formation) was assessed by scoring 800 renal cortical tubules in randomly selected fields for each subject. ${ }^{20}$ All scoring was performed on blinded slides by two independent observers.

\section{Evaluation of Fibrin and Fibrinogen Depositions}

The sections embedded in paraffin were stained with phosphotungstic acid hematoxylin (PTAH) reagent and were examined for the development of fibrin formation. They were stained with polyclonal sheep anti-human fibrinogen antibody (Acris Antibodies $\mathrm{GmbH}$, Herford, Germany), followed by biotinylated rabbit anti-sheep IgG antibody (Vector, Burlingame, CA) and avidin-biotinylated horseradish peroxidase complex (Vectastain Elite ABC kit, Vector, Burlingame, CA). The extent of fibrin deposition for each subject, which was indicated as PTAH- or fibrinogenpositive areas, was assessed as a percentage of the total glomerular area by examination of all glomeruli that were cut at their vascular pole in the section analyzed. Blood components were excluded in PTAH staining. The glomerular area was also stained along the outline of the Bowman's capsule, and these areas were measured using the
MetaMorph 6.3 image analysis computer program (Universal Imaging Co., West Chester, PA).

\section{Immunohistochemical Analysis of Macrophages and PAI-1}

Paraffin-embedded sections were stained with biotinylated monoclonal rat anti-mouse MAC2 antibody (Cedarlane), monoclonal rat anti-mouse macrophage marker F4/80 antibody (AbD Serotec, Oxford, UK), or biotinylated polyclonal rabbit anti-mouse plasminogen activator (PAI)-1 antibody (Abcam, Cambridge, UK), followed by detection using an avidin-biotinylated horseradish peroxidase complex (Vectastain Elite ABC kit, Vector, Burlingame, CA) or peroxidaselabeled goat anti-rat IgG antibody [Histofine Simple Stain Max PO (Rat), Nichirei Corp., Tokyo, Japan] as appropriate. Staining was visualized with 3,3'-diaminobenzidine (Nichirei Corp.), which produces a brown color. Negative controls involved replacement of the primary antibodies with speciesmatched antibodies.

PAI-1-positive stained areas for each subject were measured in the cortical fields of 40 glomeruli, using the MetaMorph 6.3 image analysis computer program (Universal Imaging Co.). Monocytes-macrophages that were positive for Mac2 or F4/80 were counted by examination of randomly selected 20 glomeruli or 10 cortical 
fields under a microscope at $\times 200$ magnification for each subject, in a blinded manner by two independent observers, respectively.

\section{ELISA of PAI-1, MCP-1, MIP-2, and KC Expressions in Vivo}

Kidney samples were thawed over ice. A portion of each kidney was homogenized in T-PER Tissue Protein Extraction Reagent (Pierce). The homogenate was centrifuged at $10,000 \times g$ for 5 minutes at $4^{\circ} \mathrm{C}$. The supernatant was collected and used to determine total protein concentration using the bicinchoninic acid protein assay kit (Pierce). The levels of PAI-1, MCP-1, microphage inflammatory protein (MIP)-2, or keratinocyte-derived chemokine (KC) in the kidney homogenates were measured using specific enzyme-linked immunosorbent assays (ELISA) according to the instructions of the manufacturer (Innovative Research, Novi, MI; R\&D Systems, Minneapolis, $\mathrm{MN}){ }^{31}$ The results were normalized to the total protein concentration.

\section{Real-Time PCR}

Mouse kidney tissues were snap-frozen in liquid nitrogen for total mRNA isolation. To perform mRNA extraction and cDNA synthesis, we used the RNeasy Mini Kit and the QuantiTect Reverse Transcriptional Kit (Qiagen, Hilden, Germany), according to the manufacturer instructions. Realtime PCR analysis was performed with an Applied Biosystems Prism 7500HT sequence detection system using TaqMan gene expression assays according to the manufacturer specifications (Applied Biosystems, Foster City, CA). TaqMan probes and primers for PAI-1 (Serpine; Mm00435860_m1), MK, ${ }^{16}$ MCP-1 (Ccl2; Mm00441242_m1), MIP-2 (Cxcl2; Mm00436450_m1), KC (Cxcl1; Mm04207460_m1), tPA (Plat; Mm00476931_m1), uPA (Plau; Mm00447054_m1), TGF- $\beta$ (tgf- $\beta$; Mm01178820_m1), renin, ${ }^{16}$ angiotensinogen $(\mathrm{AGT})^{16}$ and angiotensin-converting enzyme (ACE), ${ }^{16}$ and glyceraldehyde-3-phosphate dehydrogenase (GAPDH) (Gapdh; Mm99999915_g1) were used. Amplification data were analyzed with Applied Biosystems Sequence Detection software version 1.3.1.

\section{Laser Microdissection}

Formalin-fixed, paraffin-embedded (FFPE) kidney sections were mounted on poly-L-lysin-coated (Sigma-Aldrich), glass foil, polyethylene naphthalate slides for laser microdissection (Leica Microsystems, Wetzlar, Germany). After toluidine blue staining, glomeruli and tubulointerstitium were dissected using the LMD 7000 system (Leica Microsystems) and collected into each tubes filled with deparaffinization buffer (Qiagen). The extraction of total RNA was performed with the RNeasy FFPE kit (Qiagen). These samples were analyzed by real-time PCR.

\section{Multiplex Analysis of Serum PAI-1}

Serum PAI-1 level was measured by Milliplex map mouse serum adipokine kit (Millipore, Billerica, MA) following the manufacturer instructions. The data were analyzed in a Bioplex 200 system, using the Bioplex manager software version 6.0 (Bio-Rad, Hercules, CA).

\section{Isolation of Mouse Lung Endothelial Cells and PAI-1 Assessment in Vitro}

Endothelial cell cultures were established from the lung tissue of adult $\mathrm{Mdk}^{+/+}$or $\mathrm{Mdk}^{-/-}$mice, from which endothelial cells were isolated by conventional sieving methods using sheep anti-rat IgG Dynal beads (Invitrogen Dynal As, Oslo, Norway) coated with anti-platelet endothelial cell adhesion molecule 1 (BD Biosciences, Bedford, MA) and anti-intercellular adhesion molecule 1 (BD Biosciences) antibodies, as previously reported. ${ }^{32}$ The isolated mouse lung endothelial cells (MLECs) were identified by fluorescence-activated cell sorting, using anti-CD31 (BD Biosciences) and anti-CD102 (BD Biosciences) antibodies, and were then maintained in growth medium (Dulbecco's modified Eagle's medium containing 20\% fetal bovine serum), supplemented with endothelial cell growth stimulant (Biomedical Technologies Inc., Stoughton, MA), containing porcine heparin, nonessential amino acids, sodium pyruvate, L-glutamine, and penicillin-streptomycin (Wako Pure Chemical Industries, Ltd., Osaka, Japan).

To confirm the relationship between MK and PAI-1 expression in vitro, MLECs from $\mathrm{Mdk}^{+/+}$or $M d k^{-/-}$mice were exposed to fibrin for 72 hours as described previously. ${ }^{23}$ The fibrin gel was prepared using a biological tissue adhesive kit (Astellas Pharma Inc., Tokyo, Japan) according to the manufacturer instructions. Cell growth was synchronized and the culture media containing fetal bovine serum was changed every day. PAI-1, tPA, and uPA mRNA expression in cell lysates was then evaluated by real-time PCR analysis.

\section{Measurement of Blood Pressure}

The measurement of systolic blood pressure was performed on day 14 and day 0 , before the administration of rabbit antiGBM serum, to certify the influence of unilateral nephrectomy. Blood pressure was measured by tail cuff method. ${ }^{16}$

\section{Dot Blotting}

The blots of serum on the polyvinylidene difluoride membrane (GE Healthcare, Little Chalfont, Buckinghamshire, UK) were incubated with rabbit IgG (R\&D Systems, Minneapolis, MN) or PBS, followed by incubation with peroxidaseconjugated goat anti-rabbit IgG antibody or rabbit anti-mouse IgG antibody (Jackson Immunoresearch Laboratories, West Grove, PA), respectively. Proteins were visualized with an 
enhanced chemiluminescence detection system (GE Healthcare). The intensity of each blot was measured using the MetaMorph 6.3 image analysis computer program (Universal Imaging Co.).

\section{Statistical Analysis}

All values are expressed as mean \pm SD. Statistical analysis was performed using the unpaired, two-tailed Student's $t$-test for single comparisons or analysis of variance for multiple comparisons. Post hoc least significant difference tests were performed if the initial analysis of variance was significant. $P<0.05$ was considered to indicate statistically significant differences.

\section{Results}

Deposition of Rabbit IgG, Mouse IgG, and C3 in the Early Phases of GN

To develop a mouse model of crescentic GN, $M d k^{+/+}$ and $M d k^{-/-}$mice underwent uninephrectomy, were preimmunized with rabbit $\operatorname{IgG}$, and were then treated with rabbit anti-mouse GBM antibody (Figure 1A). No significant differences in these two genotypes were found in urinary albumin, systolic blood pressure, BUN, and pathogenic factors, such as TGF- $\beta$, PAI-1, MCP-1, renin, AGT, and ACE, before induction of accelerated Masugi nephritis (see Supplemental Figures S1 and S2). These data indicate that unilateral nephrectomy did not affect basal states of both genotypes before the induction of immune renal injury. We next evaluated the severity of the immune and inflammatory response to anti-GBM treatment in both mouse groups. In the heterologous response phase, which is affected by the injected rabbit IgG against mouse GBM, high deposition of rabbit IgG in the glomeruli was observed in both genotypes to a similar extent on day 1 (Figure 1B). Likewise, in the autologous response phase, which is a result of the reaction of mouse $\mathrm{IgG}$ with rabbit IgG preimmunized GBM, a similar degree of mouse $\mathrm{IgG}$ deposition in the GBM was observed from day 1 in the two genotypes (Figure 1B). No obvious depositions of rabbit or mouse IgG were observed in the tubulointerstitium (data not shown). In addition, $\mathrm{C} 3$ depositions in the glomeruli of both genotypes

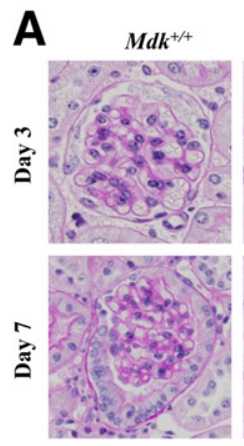

E
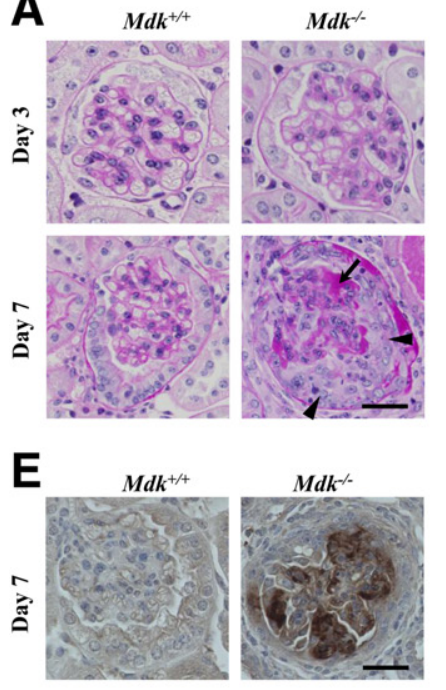

B

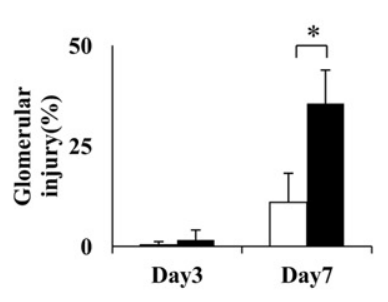

$\mathbf{F}$

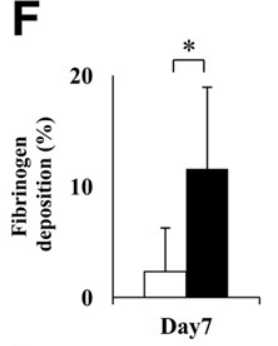

I

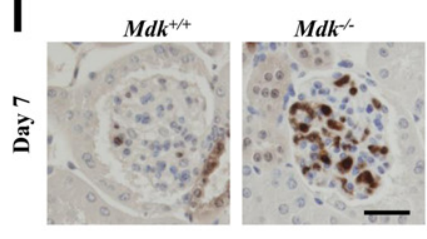

C
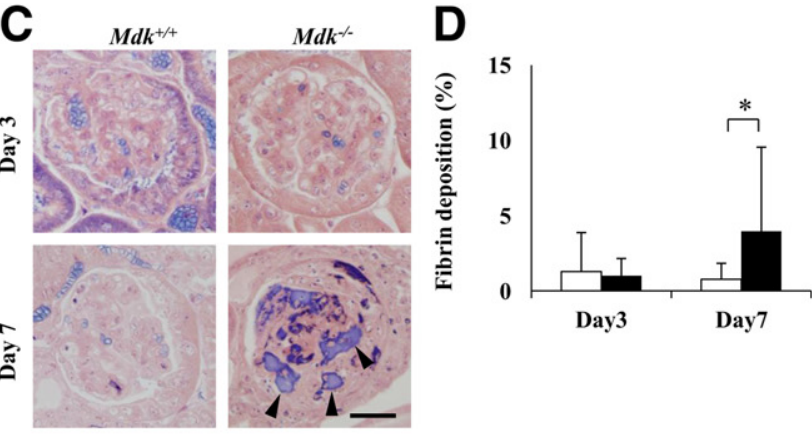

G
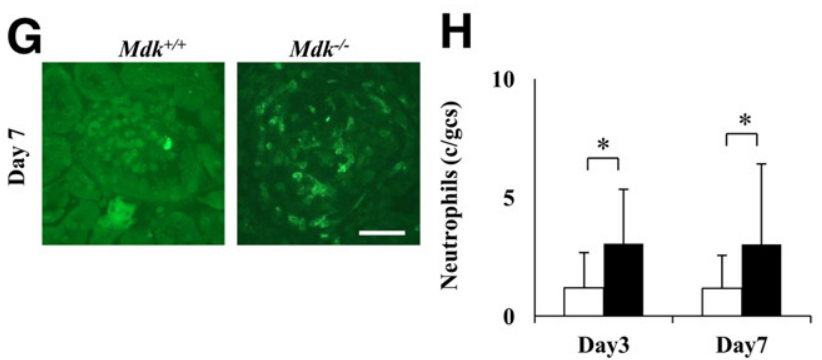

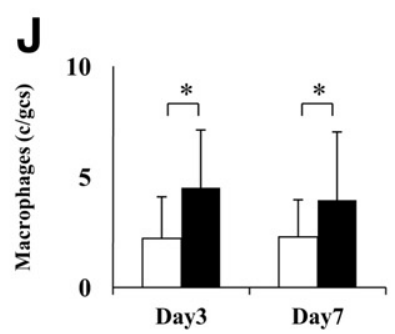

Figure 2 Glomerular histologic analysis in $\mathrm{Mdk}^{+/+}$and $\mathrm{Mdk}^{-/-}$mice. A: A glomerulus on day 3 and 7 that was stained with PAS. Arrowhead, crescent; arrow, necrosis. B: Quantitative analysis of glomerular injury, including crescent formation and necrotizing lesions. C: PTAH-stained glomeruli. Arrowheads, fibrin deposition (blue). D: Quantitative analysis of fibrin deposition. E: Immunohistochemical staining of fibrinogen deposition on day 7. F: Quantitative analysis of fibrinogen deposition. G: Immunofluorescent staining of neutrophils on day 7 . H: The number of neutrophils infiltrated into the glomerulus. c/gcs, count per glomerular crosssection. I: Immunohistochemical staining of macrophages on day 7. J: The number of macrophages infiltrated into the glomerulus. Data are means $\pm \mathrm{SD}$. White bars, $M d k^{+/+}$mice; black bars, $M d k^{-1-}$ mice. Scale bars: $25 \mu \mathrm{m}(\mathbf{A}, \mathbf{C}, \mathbf{E}, \mathbf{G}$, and $\mathbf{I})$. ${ }^{\star} P<0.01 . n=7$ per group. 
showed a similar distribution to those of mouse $\mathrm{IgG}$ (Figure 1B). Titers of circulating mouse anti-rabbit IgG were evaluated by dot blotting. $M d k^{+/+}$mice showed a little increase in mouse anti-rabbit IgG compared with $M d k^{-/-}$ mice (see Supplemental Figure S3).

\section{Marked Glomerular Injury in $\mathrm{Mdk}^{-/-}$Mice}

Renal function in these treated mice was then evaluated based on BUN values. BUN levels had increased in both genotypes to a similar extent by day 3 , but by day 7 , the BUN levels in $M d k^{-/-}$mice were significantly increased compared with those in the $M d k^{+/+}$mice (Figure 1C). Both groups had a marked rise of urinary albumin by day 7 , which was more severe in $M d k^{-/-}$than $M d k^{+/+}$mice (Figure 1D).

To determine the role of MK in crescentic GN, we next compared the degree of glomerular damage in the treated $M d k^{+/+}$and $M d k^{-/-}$mice. No obvious crescent formation was observed on day 3 in either genotype (Figure 2A). However, glomerular injuries, including crescent formation and necrotizing lesions, gradually became more severe during the next 4 days in both groups but by day 7 were
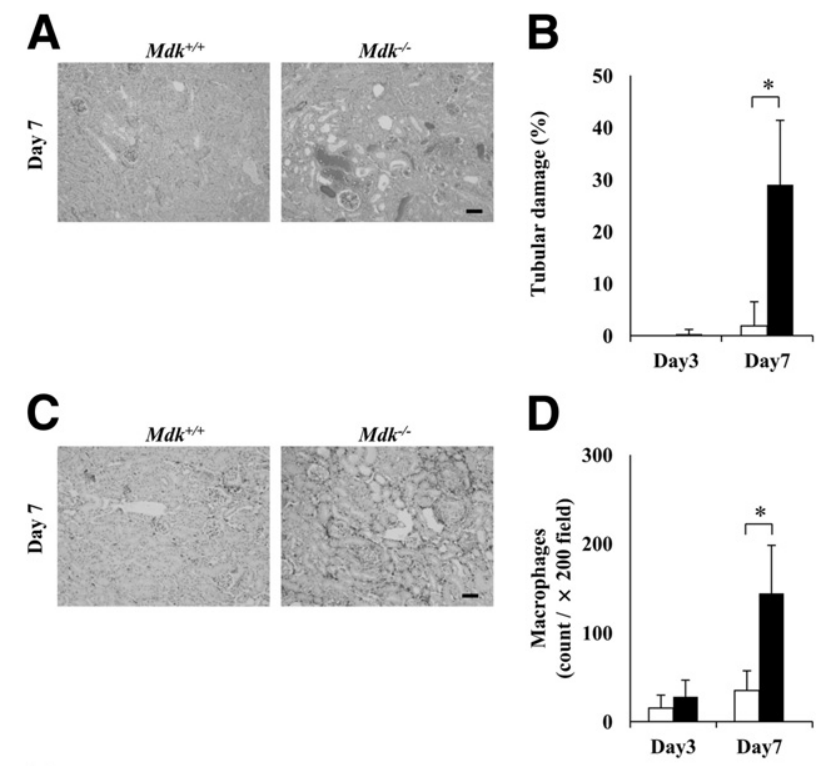

$\mathbf{E}$

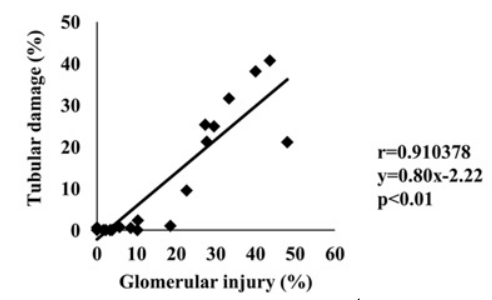

Figure 3 Tubulointerstitial injury in $\mathrm{Mdk}^{+/+}$and $\mathrm{Mdk}^{-/-}$mice. A: PAS staining of the tubulointerstitium on day 7 is shown. B: Quantitative analysis of tubular damage. C: Macrophage infiltration into the tubulointerstitium. D: The numbers of macrophages infiltrated into the renal cortex. E: Correlation of glomerular injury (crescent formation) with tubular damage. Data are means $\pm \mathrm{SD}$. White bars, $\mathrm{Mdk}^{+/+}$mice; black bars, $\mathrm{Mdk}^{-/-}$ mice. Scale bars: $50 \mu \mathrm{m}(\mathbf{A}$ and $\mathbf{C}) .{ }^{*} P<0.01 . n=7$ per each group. observed to a higher extent in $M d k^{-/-}$mice than in $M d k^{+/+}$ mice (Figure 2, A and B). Eventually, the glomeruli of $M d k^{-/-}$mice became obsolescent (data not shown).

Fibrin and fibrinogen depositions, which are strongly involved in the development of crescent formation, were also prominent in $\mathrm{Mdk}^{-/-}$mice but not in $\mathrm{Mdk}^{+/+}$mice on day 7 (Figure 2, C and E). Quantitative evaluation of this deposition with the aid of an image analysis program indicated that the profile of fibrin deposition was consistent with that of glomerular injury (Figure 2, B, D, and F). The number of both infiltrating macrophages and neutrophils was significantly increased in $M d k^{-/-}$mice at day 3 and day 7 compared with the number in $M d k^{+/+}$mice (Figure 2, G-J). These recruitments into the glomerulus had occurred on day 3 ahead of glomerular injury (Figure 2, B, $\mathrm{H}$, and $\mathbf{J}$ ).

\section{$M d k^{-/-}$Mice Show More Severe Tubulointerstitial Injury Than $M d k^{+/+}$Mice}

PAS staining showed that $M d k^{-/-}$mice exhibited more severe tubular damage than $M d k^{+/+}$mice on day 7 , as evidenced by the presence of atrophic tubules, condensation of tubular nuclei, and cast formation (Figure 3, A and B). In contrast, $M d k^{+/+}$mice showed only a little tubular atrophy (Figure 3A). Interstitial fibrosis, as assessed by collagen III expression, was evident in $M d k^{--}$mice on day 7 (data not shown). PAS staining also revealed a marked infiltration of inflammatory cells in addition to tubular atrophy in $M d k^{-1-}$ mice on day 7 (Figure 3A). Macrophage infiltration is a key event for the pathogenesis of renal damage. ${ }^{33}$ Similar to these findings, macrophage infiltration in the interstitium was prominent in $M d k^{-/-}$mice on day 7 (Figure 3, C and D). Tubular damage positively correlated with glomerular injuries (Figure 3E), suggesting that glomerular injuries, at least in part, caused the tubulointerstitial damage that was observed in $M d k^{-/-}$mice in the present study.

\section{PAI-1 Expression Markedly Increases in $\mathrm{Mdk}^{-/-}$Mice Compared with $\mathrm{Mdk}^{+/+}$Mice}

MK has been implicated in the regulation of PAI-1 activation in vitro in bovine endothelium. ${ }^{24}$ Because PAI- 1 is involved in the pathogenesis of crescentic GN, we investigated the relationship between MK and PAI-1 expression in this disease. To this end, we determined PAI- 1 expression in progressive GN of $M d k^{+/+}$or $M d k^{-/-}$mice. Compared with $M d k^{+/+}$mice, the PAI-1 protein expression in $M d k^{-/-}$ mice was more strongly expressed in components of the crescent and glomerular endothelium but not in the tubulointerstitium (Figure 4, A and B). This induction in the glomeruli of $M d k^{-/-}$mice was significantly increased compared with $M d k^{+/+}$mice on day 3 before crescent formation. This profile was consistent with the profiles of macrophage and neutrophil recruitments (Figure 2, H, J, and B). PAI-1 protein and mRNA expressions in the whole 

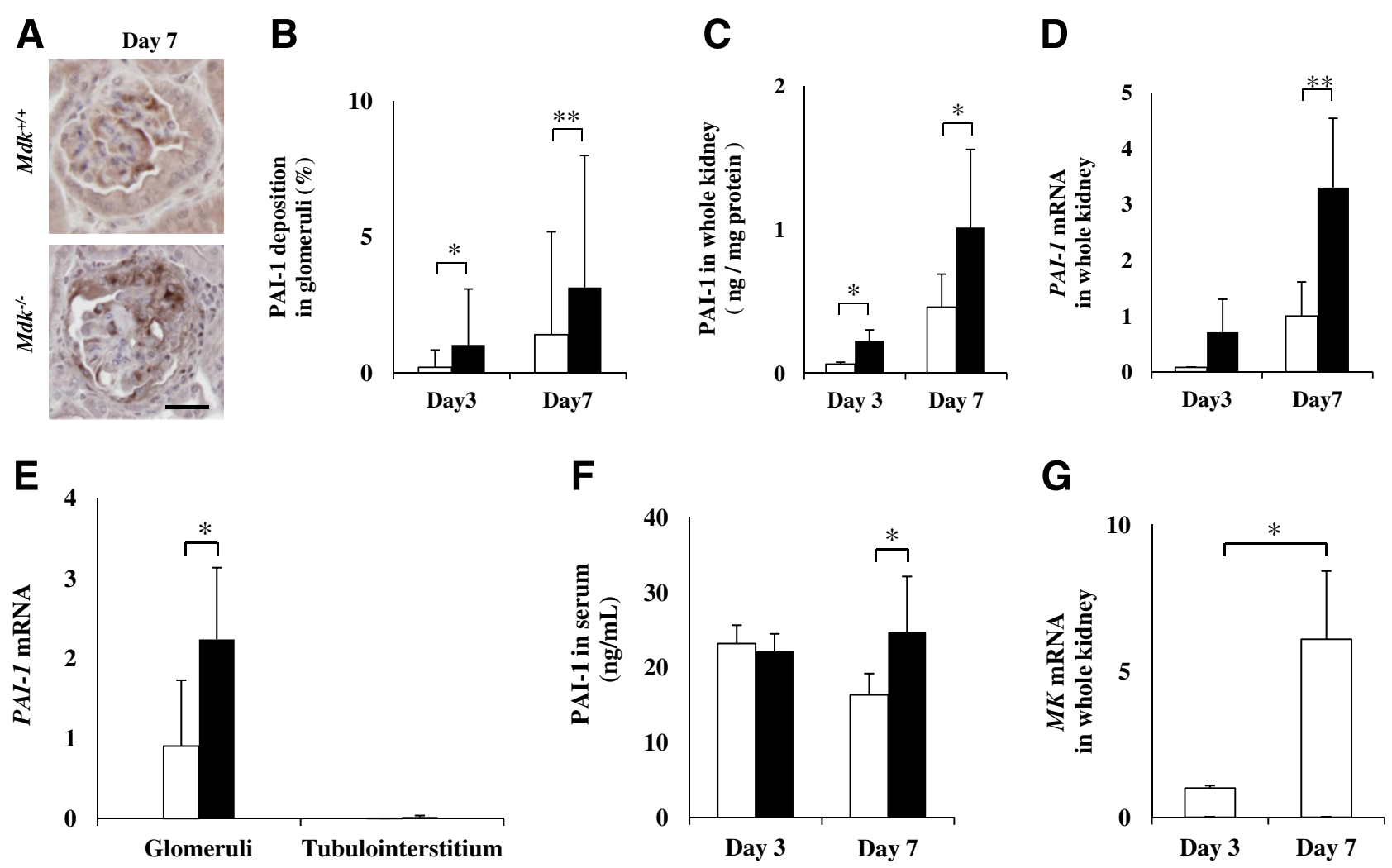

Figure 4 PAI-1 expression in $\mathrm{Mdk}^{+/+}$and $\mathrm{Mdk}^{-/-}$kidneys. A: Immunohistochemical analysis of PAI-1 expression. PAI-1 was expressed mainly in formed crescents and in glomerular endothelial cells in $\mathrm{Mdk}^{-1-}$ mice on day 7. Scale bar $=25 \mu \mathrm{m}$. B: Quantitative analysis of PAI-1 protein expression in glomeruli. C: PAI-1 protein expression in whole kidneys. D: PAI-1 mRNA expression in whole kidneys, examined using real-time PCR. Expression levels were normalized to that of GAPDH. E: PAI-1 mRNA expression in glomeruli and tubulointerstitium isolated by laser microdissection. F: Serum PAI-1 values. G: MK mRNA expression in whole kidneys, examined using real-time PCR. Data are means \pm SD. White bars, $M d k^{+/+}$mice; black bars, $M d k^{-/-}$mice. ${ }^{*} P<0.05,{ }^{* *} P<0.01 . n=7$ per group.

kidney were strikingly elevated in $M d k^{-/-}$mice compared with the $M d k^{+/+}$mice (Figure 4, C and D). We also found a significantly higher expression of PAI-1 mRNA in the glomeruli of $M d k^{-/-}$mice (Figure 4E), which was consistent with the data in Figure 4, A and B. Serum PAI-1 values tended to be gradually decreased in $M d k^{+/+}$mice on day 7 , when these levels of $M d k^{+/+}$mice were significantly lower than those of $M d k^{-/-}$mice (Figure 4F). Interestingly, MK mRNA was strongly induced in $M d k^{+/+}$mice during the experimental period (Figure 4G).

We next determined the induction of chemokines in $M d k^{+/+}$ and $M d k^{-/-}$mice. MCP-1 protein expression was more prominent in $M d k^{-/-}$mice than in $M d k^{+/+}$mice during the progression of GN (Figure 5A). A similar tendency was observed for MIP-2 and KC (Figure 5, C-F). These data suggest that the inductions of these chemokines that are responsible for the recruitment of inflammatory cells during the progression of GN were not due to the activity of MK.

\section{PAI-1 Expression in $\mathrm{Mdk}^{+/+}$or $\mathrm{Mdk}^{-/-}$MLECs}

To determine whether the difference in PAI-1 expression between $M d k^{+/+}$and $M d k^{-/-}$mice was due to the presence or absence of MK, we next examined PAI-1 mRNA expression in $M d k^{+/+}$or $M d k^{-/-}$MLECs exposed to fibrin. MLECs that were isolated using the double-selection method described in Materials and Methods showed a cobblestone appearance similar to the typical morphologic features of vascular endothelium (Figure 6A). Fluorescence-activated cell sorting analysis indicated that $97.5 \%$ of these isolated MLECs expressed both CD31 and CD102 (Figure 6B). A fibrin layer was dissolved to a greater extent by MLECs derived from $M d k^{+/+}$mice than by those derived from $M d k^{-1-}$ mice (Figure 6C). Incubation with fibrin led to an increase in PAI-1 mRNA expression at 72 hours in the $M d k^{-1-}$ MLECs but not to an increase in tPA or uPA mRNA (Figure 6D). Exposure of these cells to fibronectin that was used as a negative control showed no change in the level of these mRNAs (data not shown), which was consistent with a previous report. ${ }^{23}$

\section{Discussion}

The pathophysiologic roles of MK are diverse, ranging from acute kidney injury to chronic kidney disease that is accompanied by hypertension, ischemia, and diabetes. ${ }^{16,20-22}$ Our previous in vivo studies revealed that MK plays deleterious roles in both glomerular damage and tubulointerstitial injury through the recruitment of inflammatory cells. In contrast to these previous results, the present study demonstrated that, 

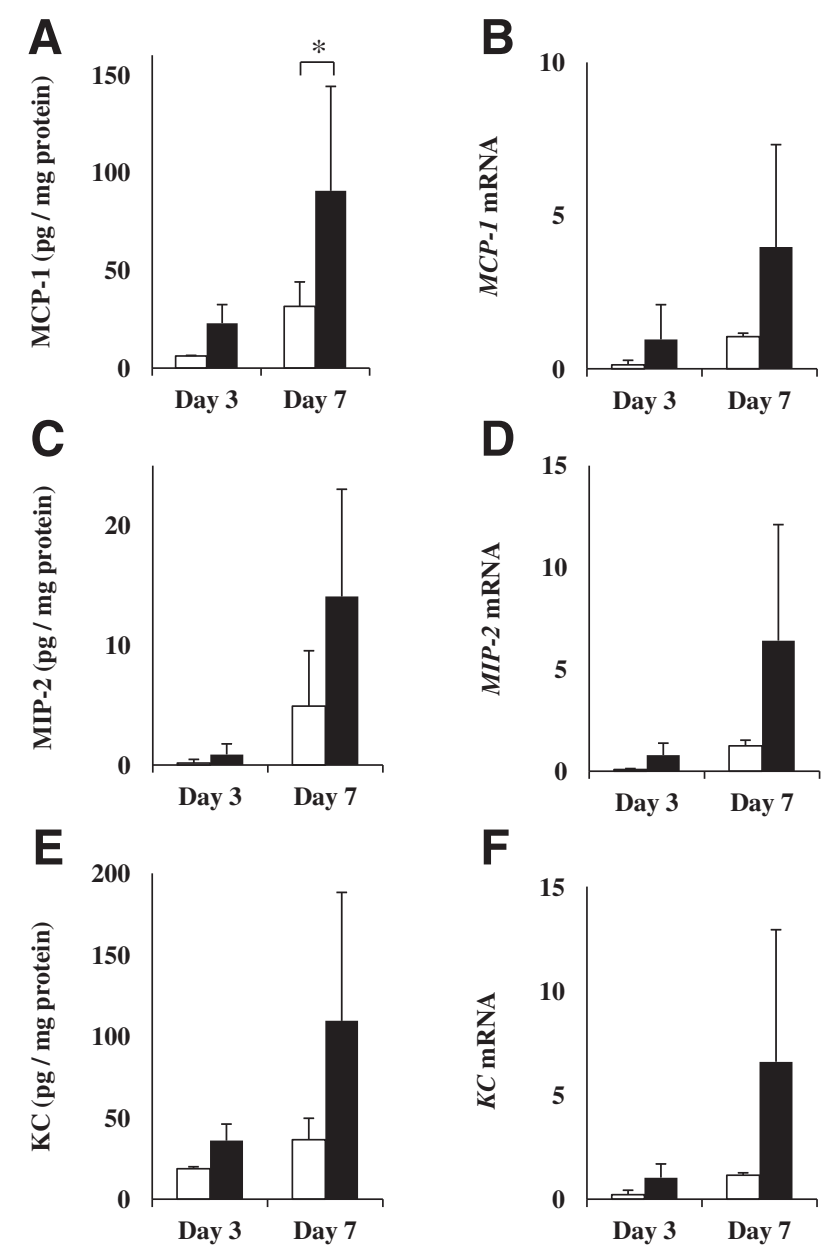

Figure $5 \mathrm{MCP}-1, \mathrm{MIP}-2$, and $\mathrm{KC}$ expressions in $\mathrm{Mdk}^{+/+}$and $\mathrm{Mdk}^{-/-}$ kidneys. MCP-1 protein (A) and mRNA (B), MIP-2 protein (C) and mRNA (D), and KC protein (E) and mRNA (F) expression in whole kidneys, assayed using an ELISA or real-time PCR, respectively. Expression levels were normalized to those of GAPDH. Data are means \pm SD. White bars, $\mathrm{Mdk}^{+/+}$ mice; black bars, $\mathrm{Mdk}^{-/-}$mice. ${ }^{\star} P<0.05 . n=7$ per group.

despite the lack of any significant differences in autologous or heterologous reactions, $M d k^{-/}$mice unexpectedly showed more necrotizing glomerular injuries than $\mathrm{Mdk}^{+/+}$mice in accelerated Masugi nephritis in mice. This difference between the 2 genotypes became apparent after 7 days. The profile of glomerular injury was consistent with crescent formation and fibrin deposition. Furthermore, greater tubular damage, together with interstitial collagen III deposition, were found in $M d k^{-/-}$mice than in $M d k^{+/+}$mice (data not shown) and positively correlated with glomerular damage. The combined data indicate that MK may play a protective role against crescentic GN and that the pathologic features of crescentic GN may be attributed to an imbalance in the coagulation-fibrinolysis system.

The formation of intravascular fibrin that is accompanied by capillary endothelial dysfunction promotes macrophage infiltration, thereby leading to interruption of the GBM and leakage of its various contents, including fibrin, red blood cells, extracellular matrix, and inflammatory cells. In this setting, macrophage recruitment might be the first step in a critical cellular circuit that is important for the development of crescent formation. Indeed, ablation of macrophages halts the progression of crescentic GN. ${ }^{34}$ In addition to its stabilization of the fibrin net, PAI-1 itself also functions as a chemoattractant for monocytes and leukocytes. ${ }^{2,35}$ In this context, it is noteworthy that both macrophages and PAI-1 enhance fibrin formation and that fibrin is also a strong inducer of macrophage infiltration and endothelial dysfunction. ${ }^{10}$ Consistent with the idea, we demonstrated that a significant increase in PAI-1 expression and macrophage infiltration occurred in $\mathrm{Mdk}^{-/-}$mice as early as 3 days (Figure 2, J and B). To further support our data, a previous elegant study demonstrated that a greater number of crescents were formed, along with extensive fibrin deposits and macrophage infiltration, in PAI-1-overexpressing mice than in PAI-1-deficient mice in experimental models of antiGBM crescentic GN. ${ }^{10}$ Indeed, high PAI-1 expression is detected both in areas of glomerular necrosis and in crescents in progressive GN. ${ }^{36,37}$ In particular, parietal epithelial cells and glomerular endothelial cells appeared to express PAI-1. In line with the in vivo data, we also proved that primarycultured endothelial cells derived from $\mathrm{Mdk}^{-/-}$mice exhibited more PAI-1 mRNA on fibrin challenge and less fibrinolysis than cells from $M d k^{+/+}$mice. In contrast, the expression of plasminogen activators, such as TPA and uPA, was not affected. Although we have not verified a direct relationship among MK, PAI-1, and crescentic GN, our
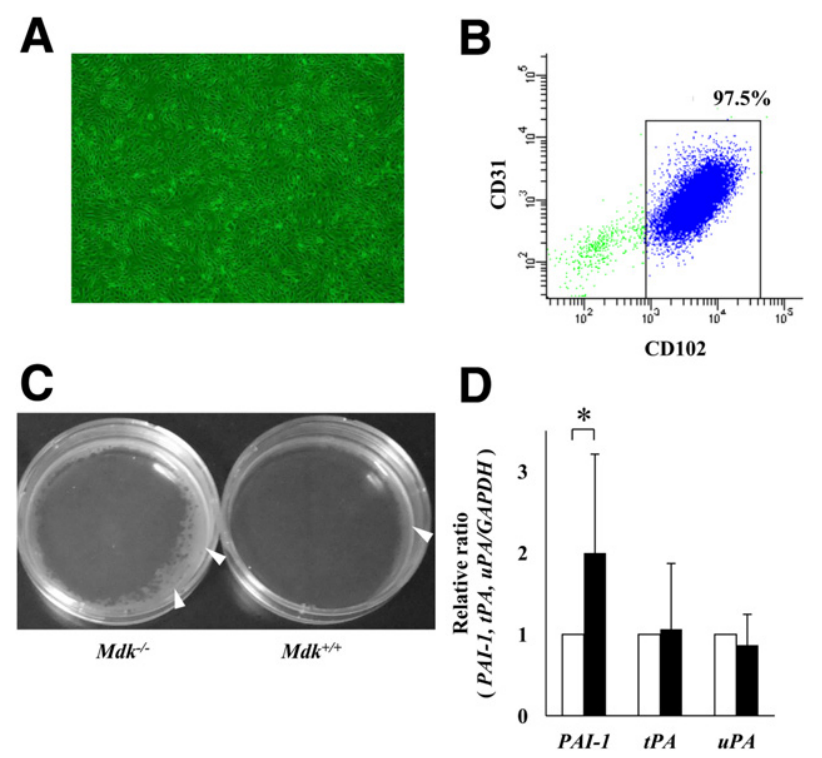

Figure 6 Fibrinolytic effect of MK through the regulation of PAI-1 in primary-cultured $\mathrm{Mdk}^{+/+}$and $M d k^{-/-}$MLECs. A: Morphologic analysis of primary-cultured MLECs derived from $M d k^{-/-}$mice, assayed using phase contrast microscopy. B: Flow cytometric analysis of CD31 and CD102 expression in isolated MLECs. C: Visible dissolution of a fibrin layer by $\mathrm{Mdk}^{+/+}$ or $\mathrm{Mdk}^{-1-}$ MLECs. Arrowhead, residual fibrin layer. D: PAI-1, tPA, and uPA mRNA expression in $\mathrm{Mdk}^{+/+}$or $\mathrm{Mdk}^{-/-}$MLECs after 72 hours of incubation in the presence of fibrin. Expression levels were normalized to that of GAPDH. Data shown are the mean and SD of four independent experiments. White bars, $M d k^{+/+}$mice; black bars, $M d k^{-/-}$mice. ${ }^{*} P<0.05$. 
study indicates that these three factors are closely associated. Further studies to examine whether the MK-PAI-1 axis directly and predominantly leads to renal inflammation, including crescentic GN, will provide a new insight into the pathogenesis of human kidney diseases.

There is evidence that the level of PAI-1 expression might help determine the severity of renal injuries in human crescentic GN. ${ }^{3}$ PAI-1 is induced in response to various stimuli, including high ambient glucose, TGF- $\beta$, oxidative stress, and angiotensin II. However, to date, the precise mechanism by which PAI-1 is suppressed in the kidneys has not yet been completely elucidated. Our data suggested that MK may be a candidate reagent for the prevention or delay of rapidly progressive GN.

\section{Acknowledgments}

We thank Norihiko Suzuki, Naoko Asano, and Yuriko Sawa for their excellent technical assistance and Hitomi Aoyama for secretarial assistance.

\section{Supplemental Data}

Supplemental material for this manuscript can be found at http://dx.doi.org/10.1016/j.ajpath.2012.10.016.

\section{References}

1. Eddy AA, Fogo AB: Plasminogen activator inhibitor-1 in chronic kidney disease: evidence and mechanisms of action. J Am Soc Nephrol 2006, 17:2999-3012

2. Hertig A, Rondeau E: Role of the coagulation/fibrinolysis system in fibrin-associated glomerular injury. J Am Soc Nephrol 2004, 15: 844-853

3. Ha H, Oh EY, Lee HB: The role of plasminogen activator inhibitor 1 in renal and cardiovascular diseases. Nat Rev Nephrol 2009, 5: 203-211

4. Roelofs JJ, Teske GJ, Bonta PI, de Vries CJ, Meijers JC, Weening JJ, van der Poll T, Florquin S: Plasminogen activator inhibitor-1 regulates neutrophil influx during acute pyelonephritis. Kidney Int 2009, 75: 52-59

5. Lee EA, Seo JY, Jiang Z, Yu MR, Kwon MK, Ha H, Lee HB: Reactive oxygen species mediate high glucose-induced plasminogen activator inhibitor-1 up-regulation in mesangial cells and in diabetic kidney. Kidney Int 2005, 67:1762-1771

6. Jiang Z, Seo JY, Ha H, Lee EA, Kim YS, Han DC, Uh ST, Park CS, Lee HB: Reactive oxygen species mediate TGF-beta1-induced plasminogen activator inhibitor-1 upregulation in mesangial cells. Biochem Biophys Res Commun 2003, 309:961-966

7. Liao H, Hyman MC, Lawrence DA, Pinsky DJ: Molecular regulation of the PAI-1 gene by hypoxia: contributions of Egr-1, HIF-1alpha, and C/EBPalpha. FASEB J 2007, 21:935-949

8. Yoshimoto T, Fukai N, Sato R, Sugiyama T, Ozawa N, Shichiri M, Hirata Y: Antioxidant effect of adrenomedullin on angiotensin II-induced reactive oxygen species generation in vascular smooth muscle cells. Endocrinology 2004, 145:3331-3337

9. Yoshimoto T, Gochou N, Fukai N, Sugiyama T, Shichiri M, Hirata Y: Adrenomedullin inhibits angiotensin II-induced oxidative stress and gene expression in rat endothelial cells. Hypertens Res 2005, 28: $165-172$
10. Kitching AR, Kong YZ, Huang XR, Davenport P, Edgtton KL, Carmeliet P, Holdsworth SR, Tipping PG: Plasminogen activator inhibitor-1 is a significant determinant of renal injury in experimental crescentic glomerulonephritis. J Am Soc Nephrol 2003, 14: $1487-1495$

11. Kadomatsu K, Muramatsu T: Midkine and pleiotrophin in neural development and cancer. Cancer Lett 2004, 204:127-143

12. Unoki K, Ohba N, Arimura H, Muramatsu H, Muramatsu T: Rescue of photoreceptors from the damaging effects of constant light by midkine, a retinoic acid-responsive gene product. Invest Ophthalmol Vis Sci 1994, 35:4063-4068

13. Yoshida Y, Goto M, Tsutsui J, Ozawa M, Sato E, Osame M, Muramatsu T: Midkine is present in the early stage of cerebral infarct. Brain Res Dev Brain Res 1995, 85:25-30

14. Yoshida Y, Ikematsu S, Moritoyo T, Goto M, Tsutsui J, Sakuma S, Osame M, Muramatsu T: Intraventricular administration of the neurotrophic factor midkine ameliorates hippocampal delayed neuronal death following transient forebrain ischemia in gerbils. Brain Res 2001, 894:46-55

15. Takada J, Ooboshi H, Ago T, Kitazono T, Yao H, Kadomatsu K, Muramatsu T, Ibayashi S, Iida M: Postischemic gene transfer of midkine, a neurotrophic factor, protects against focal brain ischemia. Gene Ther 2005, 12:487-493

16. Hobo A, Yuzawa Y, Kosugi T, Kato N, Asai N, Sato W, Maruyama S, Ito Y, Kobori H, Ikematsu S, Nishiyama A, Matsuo S, Kadomatsu K: The growth factor midkine regulates the renin-angiotensin system in mice. J Clin Invest 2009, 119:1616-1625

17. Maruyama K, Muramatsu H, Ishiguro N, Muramatsu T: Midkine, a heparin-binding growth factor, is fundamentally involved in the pathogenesis of rheumatoid arthritis. Arthritis Rheum 2004, 50: $1420-1429$

18. Horiba M, Kadomatsu K, Nakamura E, Muramatsu H, Ikematsu S, Sakuma S, Hayashi K, Yuzawa Y, Matsuo S, Kuzuya M, Kaname T, Hirai M, Saito H, Muramatsu T: Neointima formation in a restenosis model is suppressed in midkine-deficient mice. J Clin Invest 2000, 105:489-495

19. Sato W, Kadomatsu K, Yuzawa Y, Muramatsu H, Hotta N, Matsuo S, Muramatsu T: Midkine is involved in neutrophil infiltration into the tubulointerstitium in ischemic renal injury. J Immunol 2001, 167: 3463-3469

20. Kosugi T, Yuzawa Y, Sato W, Arata-Kawai H, Suzuki N, Kato N, Matsuo S, Kadomatsu K: Midkine is involved in tubulointerstitial inflammation associated with diabetic nephropathy. Lab Invest 2007, 87:903-913

21. Kosugi T, Yuzawa Y, Sato W, Kawai H, Matsuo S, Takei Y, Muramatsu T, Kadomatsu K: Growth factor midkine is involved in the pathogenesis of diabetic nephropathy. Am J Pathol 2006, 168:9-19

22. Kato K, Kosugi T, Sato W, Arata-Kawai H, Ozaki T, Tsuboi N, Ito I, Tawada H, Yuzawa Y, Matsuo S, Kadomatsu K, Maruyama S: Growth factor midkine is involved in the pathogenesis of renal injury induced by protein overload containing endotoxin. Clin Exp Nephrol 2011, 15: 346-354

23. Kojima $\mathrm{S}$, Soga $\mathrm{W}$, Hagiwara $\mathrm{H}$, Shimonaka $\mathrm{M}$, Saito $\mathrm{Y}$, Inada $\mathrm{Y}$ : Visible fibrinolysis by endothelial cells: effect of vitamins and sterols. Biosci Rep 1986, 6:1029-1033

24. Kojima S, Muramatsu H, Amanuma H, Muramatsu T: Midkine enhances fibrinolytic activity of bovine endothelial cells. J Biol Chem 1995, 270:9590-9596

25. Nakamura E, Kadomatsu K, Yuasa S, Muramatsu H, Mamiya T, Nabeshima T, Fan QW, Ishiguro K, Igakura T, Matsubara S, Kaname T, Horiba M, Saito H, Muramatsu T: Disruption of the midkine gene (Mdk) resulted in altered expression of a calcium binding protein in the hippocampus of infant mice and their abnormal behaviour. Genes Cells 1998, 3:811-822

26. Unanue ER, Lee S, Dixon FJ, Feldman JD: Experimental glomerulonephritis. VII The absence of an autoimmune antikidney 
response in nephrotoxic serum nephritis. J Exp Med 1965, 122: $565-578$

27. Unanue ER, Dixon FJ: Experimental glomerulonephritis, V: studies on the interaction of nephrotoxic antibodies with tissue of the rat. J Exp Med 1965, 121:697-714

28. Unanue ER, Dixon FJ: Experimental glomerulonephritis, VI: the autologous phase of nephrotoxic serum nephritis. J Exp Med 1965, 121:715-725

29. Horio T: Development and fate of crescentic and granulomatous lesions in rat Masugi nephritis. Pathol Int 2001, 51:72-81

30. Steinmetz OM, Summers SA, Gan PY, Semple T, Holdsworth SR, Kitching AR: The Th17-defining transcription factor RORgammat promotes glomerulonephritis. J Am Soc Nephrol 2011, 22:472-483

31. Ishimoto T, Takei Y, Yuzawa Y, Hanai K, Nagahara S, Tarumi Y, Matsuo S, Kadomatsu K: Downregulation of monocyte chemoattractant protein-1 involving short interfering RNA attenuates hapteninduced contact hypersensitivity. Mol Ther 2008, 16:387-395

32. Lim YC, Garcia-Cardena G, Allport JR, Zervoglos M, Connolly AJ, Gimbrone MA Jr, Luscinskas FW: Heterogeneity of endothelial cells from different organ sites in T-cell subset recruitment. Am J Pathol 2003, 162:1591-1601

33. Chevalier RL, Forbes MS, Thornhill BA: Ureteral obstruction as a model of renal interstitial fibrosis and obstructive nephropathy. Kidney Int 2009, 75:1145-1152

34. Duffield JS, Tipping PG, Kipari T, Cailhier JF, Clay S, Lang R, Bonventre JV, Hughes J: Conditional ablation of macrophages halts progression of crescentic glomerulonephritis. Am J Pathol 2005, 167: $1207-1219$

35. Oda T, Jung YO, Kim HS, Cai X, Lopez-Guisa JM, Ikeda Y, Eddy AA: PAI-1 deficiency attenuates the fibrogenic response to ureteral obstruction. Kidney Int 2001, 60:587-596

36. Rondeau E, Mougenot B, Lacave R, Peraldi MN, Kruithof EK, Sraer JD: Plasminogen activator inhibitor 1 in renal fibrin deposits of human nephropathies. Clin Nephrol 1990, 33:55-60

37. Grandaliano G, Gesualdo L, Ranieri E, Monno R, Schena FP: Tissue factor, plasminogen activator inhibitor-1, and thrombin receptor expression in human crescentic glomerulonephritis. Am J Kidney J 2000, 35:726-738 